\title{
IMPACT OF THE SPECIES COMPOSITION OF A TREE STAND ON THE NUMBER OF NATURAL YOUNG SEEDLINGS IN SELECTED NATIONAL PARKS OF THE WESTERN CARPATHIANS
}

\author{
Jerzy Skrzyszewski ${ }^{1 *}$, Maciej Pach¹, Kinga Skrzyszewska, \\ Roman Krzysztof ${ }^{2}$, Zenon Stenka ${ }^{3}$
}

\author{
${ }^{1}$ Department of Ecology and Silviculture, Faculty of Forestry, \\ University of Agriculture in Krakow, al. 29 Listopada 46, 31-425 Krakow \\ ${ }^{2}$ Limanowa Forest Division, ul. Kopernika 3, 34-600 Limanowa \\ ${ }^{3}$ Office of Forest Management and Geodesy, Gdynia branch, \\ ul. Świętojańska 44, 81-393 Gdynia
}

\section{ABSTRACT}

The study uses data from 2288 sample plots established in forest stands with limited human interference in the Carpathian National Parks (the Bieszczady, Magura, Gorce, and Babia Góra National Parks). The research area is characterised by a full habitat spectrum of lower montane zone forest stands of the western arc of the Carpathians. The aim of the study was to verify the hypothesis that among trees forming a stand, species that have a positive or negative impact on the number of natural young seedlings of firs, beeches, spruces and sycamores can be separated.

A positive impact of basal area of potential seed trees of a given species on the number of the species' regeneration was found. A significantly greater impact of basal area of seed trees on the number of renewals of a given species was observed in the case of spruce and sycamore than in the case of shade-tolerant species as fir and beech. However, the increase in basal area of all trees of a given species (with the diameter at breast height, or $\mathrm{DBH}$, from $7 \mathrm{~cm}$ upwards), limited the number of natural young seedlings of this species.

*凶rlskrzys@cyf-kr.edu.pl 
The impact of species growing in the stand on the number of natural young seedlings depended on the scale $(11,5.3$ meters) in which the phenomenon was observed.

The increase in the basal area of pioneer species in the stand limited the number of natural young seedlings of firs, beeches and spruces, while favouring of sycamores. More numerous regeneration of spruce was observed in the immediate vicinity of sycamore (3-metre scale), but on the 11- and 5-metre scale the occurrence of sycamores acted the opposite. Spruce growing in the stand had a positive impact on the number of natural young seedling of all analysed species (especially on a 5-metre scale), in contrast to fir which showed no clear positive relationship on any scale with the natural young seedlings, of its own or other species. The presence of beech in the stand favoured the renewal of fir and spruce, beech had this kind of impact for natural young seedlings of spruce.

The occurrence in a stand of tree species that positively impact the development of natural young seedlings of a given tree species does not guarantee their renewal. However, if favourable conditions for renewal occur, then in places of occurrence of species positively impacting the renewal of a given species will be more numerous.

\section{KEYWORDS}

natural forest regeneration $\bullet$ mixed forests $\bullet$ fir $\bullet$ spruce $\bullet$ beech $\bullet$ sycamore $\bullet$ Abies - Fagus $\bullet$ Picea $\bullet$ Acer $\bullet$ facilitation

\section{Introduction}

Natural forest regeneration is a basic condition for maintaining forest continuity. The regeneration process depends, among other things, on the habitat conditions understood as a set of abiotic factors (climate and soil). The reproductive success of a species also depends on the biotic factors of an environment, such as the co-occurrence of other tree species (Jaworski 2011), groundstory vegetation (Bernadzki 2008), fungi growing in an above-ground environment, and soil microbiota (Cline et al. 2007, Grebenc et al. 2009). The impact of species of plants is associated with the formation of light conditions (Jaworski 2011) and organic soil litter, which, together with root exudation and allelopathic interaction (Becker and Drapier 1985) modifies the chemistry of the top organic levels of soil (Paluch and Gruba 2012, Bigelow and Canham 2015) but it can also be a physical barrier for seedling (germinants) development (Bens et al. 2006, Buck and St Clair 2014). The impact of pathogens and symbionts depends on their number and species diversity, species characteristics and environmental conditions that favour or limit their development. These factors determine the availability of a regeneration niche. The complex of these factors and the interactions between them are connected to the features associated with the tree species: mast seeding years, content of nutrients in seeds, as well as biological features and life strategies of the species (Brzeziecki 2000, Jaworski 2011). Also, human influence is significant: direct as a result of silvicultural practices and indirect through the impact of industry or maintenance of game population (Szukiel 1982). All listed components of reproductive success or failure occur together and are characterised by different scales of spatial impact. Humans can only 
slightly modify the habitat conditions but have a decisive impact on stand characteristics such as density or species composition. Despite the extensive literature on the subject, the problem of identifying the factors determining the occurrence and number of regenerations is still valid. The measurement data used in this work come from four selected Carpathian national parks. The research area is characterised by a full habitat spectrum of lower montane zone forest stands of the western arc of the Carpathians.

The number of naturally regenerated firs, beeches, spruces, and sycamores was analysed. The first three species have dominant economic importance in the Carpathian forests. Sycamore mainly acts as an admixture species. However, its wood is valued and it is considered a species whose potential will increase as a result of climate changes (Szwagrzyk 2014). Species included in the study are characterised by different biology and habitat requirements.

Fir is a shade-tolerant species. Investigations conducted for a number of years indicate the adverse impact of single-species stands of fir trees on the possibility of regeneration of this species under the mature stand (Jaworski 1973). The factors limiting the chances of germinants survival until the younger seedling phase are: mass germinants infestation by fungi causing damping-off in conditions of intense precipitation (Paluch and Stępniewska 2012), autotoxicity, restrictive effect of high concentration of manganese and aluminum (Jaworski 1973, 2011), strongly developed groundstory vegetation (Bernadzki 2008). The reproductive success of fir is thought to be increased by the vicinity of spruce (Paluch and Jastrzębski 2013, Paluch et al. 2016). In the fertile beech habitats of the Carpathians, fir regenerates better in beech stands, and beech regenerates better under fir stands (Sugiero 2005). Paluch et al. (2016) found that the presence of beech, especially on a single tree scale, promotes fir regeneration. There is a greater efficiency of fir regeneration in stands where species that acidify the top soil levels and reduce the rate of decomposition of organic matter are present, e.g. spruce (Jaworski 1973, Hofmeister et al. 2008, Paluch and Jastrzębski 2013). In addition, species with an transparent crown, such as pine and larch create favourable light conditions (Ambroży 2002, Dobrowolska 2013). Beech, being a shade-tolerant species, but less tolerant than fir, is also an expansive species widely regenerating itself even in single-species beech stands of medium age classes. In conditions of wide self-sowing and favourable light conditions, it can effectively compete, especially with shade-intolerant sycamore (Przybylska and Kucharzyk 1999, Bartkowicz et al. 2008).

Spruce is characterised by a very high seed dispersion and high regeneration dynamics (Jaworski 2011). It is moderately shade-tolerant, develops a horizontal, shallow root system and in conditions of good access to light and shallow soils it may compete with regeneration of other tree species.

Sycamore is the most shade-intolerant species among the analysed. It forms numerous regenerations that die out in the absence of light in a short period of time (Przybylska and Kucharzyk 1999, Bartkowicz et al. 2008).

The aim of the study was to verify the hypothesis that among trees forming a forest stand, it is possible to separate species that have a positive or negative impact on the number of natural young seedlings of firs, beeches, spruces, and sycamores. 


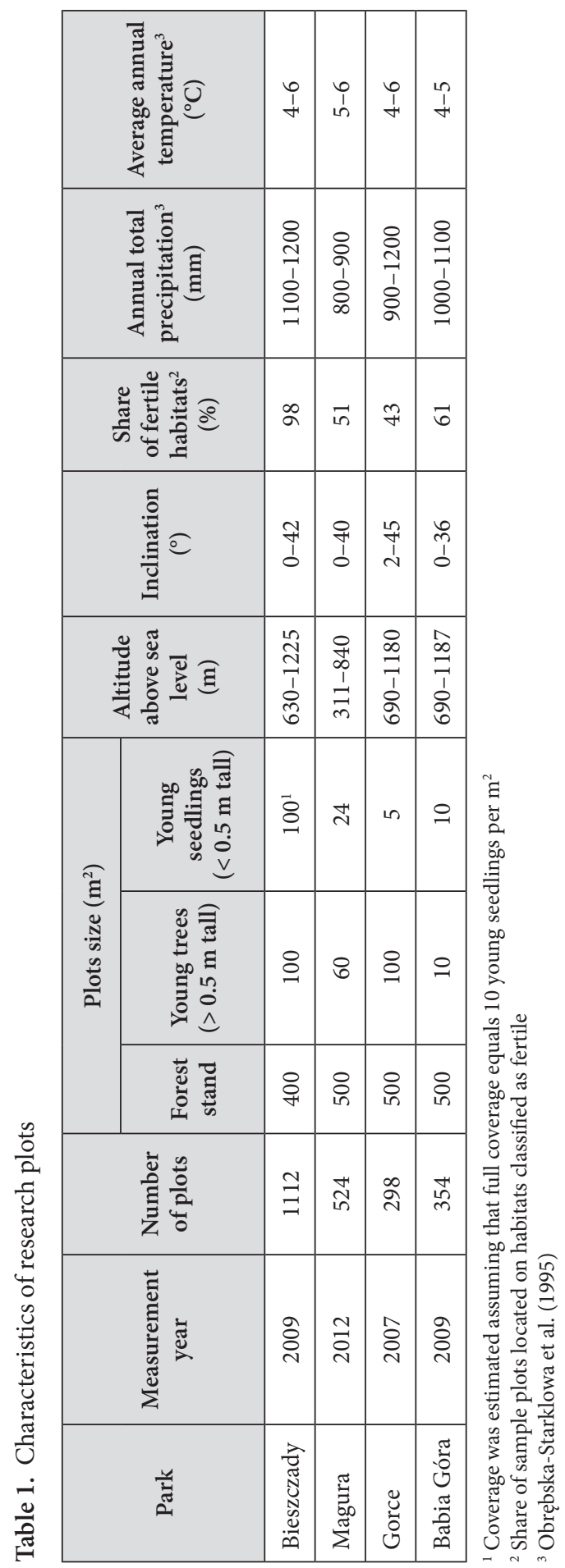




\section{Methodology}

\section{Study area}

Data used in the analysis was taken from a forest inventory conducted cyclically on permanent research plots in four Carpathian national parks. In total, the authors had data from 5,188 sample plots, set up in a grid of squares with a side length of $500 \mathrm{~m}$ (Bieszczady and Magura NP), $400 \mathrm{~m}$ (Gorce NP), and $250 \mathrm{~m}$ (Babia Góra NP) at their disposal. From the total number of plots, those that were located in the lower montane forest zone were selected. Among them, the following were rejected: areas without forest cover, artificial stands established on former agricultural lands, highly moistened forest communities within the Alno-Padion. Finally, 2288 areas were included in the calculations, assuming for the calculations measurement series from the least distant calendar years from each park. The number of plots and their features in individual national parks are given in Tables 1 and 2.

Table 2. The average share of species based on the basal area of trees on the sample plots

\begin{tabular}{|l|c|c|c|c|c|}
\hline \multirow{2}{*}{ Park } & \multicolumn{5}{|c|}{ Share of species (\%) } \\
\cline { 2 - 6 } & Abies & Fagus & Picea & Acer & Other \\
\hline Bieszczady & 9 & 74 & 6 & 6 & 5 \\
\hline Magura & 22 & 64 & $0^{*}$ & 6 & 8 \\
\hline Gorce & 23 & 51 & 24 & 1 & 1 \\
\hline Babia Góra & 9 & 30 & 57 & 2 & 2 \\
\hline
\end{tabular}

* Less than $1 \%$

\section{Data analysis}

The following habitat data were taken into account in model construction: altitude, inclination in degrees, slope exposure, habitat trophism. Habitat trophism was based on a plant community. The following were classified as less fertile: Galio-Abietetum, Abieti-Piceetum montanum, Luzulo luzuloides-Fagetum, infertile variant of Dentario glandulosae-Fagetum. Dentario glandulosae-Fagetum, Aceri-Fagetum, Lunario-Aceretum, Tilio-Carpinetum were considered fertile. In the Magura National Park, the range of plot location in the vertical gradient differed significantly from the other parks, therefore a variable was introduced into the model: the Park $\times$ Altitude interaction

The stage of selecting the stand characteristics as explanatory variables was preceded by the search for those most strongly related to the occurrence (with the logistic regression method) and the number of natural young seedlings (with the correlation method) using the following methods: selecting the best subset, assuming the Akaike criterion, and the backward elimination method (Stanisz 2007). At this stage, it was also decided 
whether to use the indicators based on the number of trees, basal area, and DBH, and whether to use direct values or competition (impact) indices.

As explanatory variables, the following were selected from the stand's features: the sum of the basal area of individual tree species with the DBH (D) from $7 \mathrm{~cm}$ and over; the sum of the basal area of potential seed trees of individual species (trees with diameter from $30 \mathrm{~cm}$ and over), the number of natural young seedlings (up to the height of $50 \mathrm{~cm}$ ) of individual tree species (in the calculation for a given species the number of its natural young seedlings was a dependent variable and the number of other species was interpreted as an expression of their competition), the number of advanced regeneration (above $50 \mathrm{~cm}$ tall, up to the DBH of $6.9 \mathrm{~cm}$ ) of all species together (interpreted as an expression of their competition). The basal area of the trees as well as the number of natural young seedlings and advanced regeneration were calculated per plot unit. The basal area was calculated for trees growing in range (scale): $3 \mathrm{~m}$ (scale of direct impact), $5 \mathrm{~m}$ (scale of a single tree) and $11 \mathrm{~m}$ (scale of forest stand) from the center of the plot. Based on the number of natural young seedlings, the number of plots was determined: with no natural young seedlings (zero), with 1 natural young seedling and below (calculated per $1 \mathrm{~m}^{2}$ ), and with above 1 natural young seedling per $\mathrm{m}^{2}$. The following species were included: fir, beech, spruce, sycamore, and others. The "other" category included 21 tree species, among which the largest share had: larch, alder, birch, willow, ash, pine, and wild cherry.

The models include location (national park) as a qualitative variable.

Calculations for a given species were made on a sample of plots on which at least one tree of this species with the $\mathrm{DBH}$ equal to or larger than $30 \mathrm{~cm}$ grew in the stand.

For the analysis of the impact of these independent variables, a generalised linear model based on the Poisson distribution with a logarithmic link function was used (Stanisz 2007).

General form of the model:

$$
g(Y)=X \cdot \beta+\varepsilon
$$

where:

$$
\begin{aligned}
& g-\text { link function, } \\
& Y-\text { vector of the dependent variable value, } \\
& X-\text { matrix of independent variable values, } \\
& \beta-\text { vector of model parameters, } \\
& \varepsilon-\text { random residual vector. }
\end{aligned}
$$

The purpose of model creation was not to predict the number of natural young seedlings but to assess the relationship between the species composition of the stand and the reproductive success of fir, beech, spruce and sycamore. The measure of reproductive success was the number of natural young seedlings of these species. Parameters of full models were analysed, taking into account all the previously mentioned explanatory variables (related to habitat and stand). In total, 12 models were created ( 4 species $\times 3$ impact radii $-3,5$ and $11 \mathrm{~m}$ ). The parameters of the variables in the models are given in Table 3. 


\begin{tabular}{|c|c|c|c|c|c|c|c|c|c|c|c|c|c|c|c|c|}
\hline \multirow{3}{*}{ 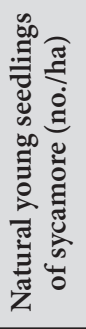 } & $\stackrel{m}{v}$ & $\begin{array}{l}\overrightarrow{\widehat{D}} \\
\stackrel{\wedge}{n}\end{array}$ & 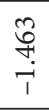 & $\underset{\text { i }}{\infty}$ & $\begin{array}{l}0 \\
\stackrel{0}{0} \\
i\end{array}$ & $\begin{array}{l}\overrightarrow{3} \\
\infty \\
i\end{array}$ & $\begin{array}{l}2 \\
\stackrel{0}{0} \\
1\end{array}$ & $\frac{\hat{\Omega}}{0}$ & $\begin{array}{l}\overrightarrow{8} \\
\dot{0}\end{array}$ & $\overrightarrow{8}$ & $\begin{array}{l}\text { ôे } \\
\delta \\
0\end{array}$ & 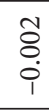 & $\begin{array}{l}2 \\
0 \\
0 \\
0 \\
i\end{array}$ & $\begin{array}{l}\text { tr } \\
0 \\
0\end{array}$ & 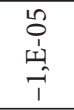 & $\begin{array}{l}\text { 옹 } \\
1 \\
1 \\
\text { m } \\
1\end{array}$ \\
\hline & $\stackrel{\text { in }}{v}$ & $\begin{array}{l}\infty \\
\infty \\
\infty \\
\infty\end{array}$ & $\stackrel{\infty}{\stackrel{\infty}{m}}$ & $\begin{array}{l}m \\
\infty \\
\infty \\
i \\
i\end{array}$ & $\begin{array}{l}+1 \\
\hat{6} \\
\dot{0} \\
1\end{array}$ & $\begin{array}{l}\stackrel{2}{\Omega} \\
\widehat{i}\end{array}$ & 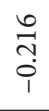 & 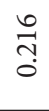 & $\overrightarrow{8}$ & $\overrightarrow{8}$ & $\overrightarrow{8}$ & $\begin{array}{l}m \\
\tilde{\delta} \\
\dot{1}\end{array}$ & $\begin{array}{l}0 \\
8 \\
\\
\end{array}$ & 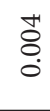 & $\begin{array}{l}\text { \& } \\
\text { 1 } \\
\text { i } \\
\text { i }\end{array}$ & 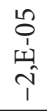 \\
\hline & $\vec{v}$ & $\underset{\infty}{\stackrel{9}{+}}$ & 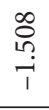 & 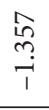 & 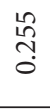 & $\begin{array}{l}\vec{b} \\
\stackrel{i}{0}\end{array}$ & $\begin{array}{l}\infty \\
\stackrel{0}{+} \\
\stackrel{1}{1}\end{array}$ & $\begin{array}{l}\infty \\
\stackrel{0}{0}\end{array}$ & ठ요 & 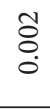 & $\begin{array}{l}+ \\
8 \\
0 \\
0 \\
1\end{array}$ & 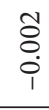 & $\begin{array}{l}\hat{8} \\
\dot{8} \\
\dot{1}\end{array}$ & 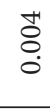 & 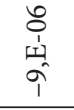 & 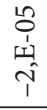 \\
\hline \multirow{3}{*}{ 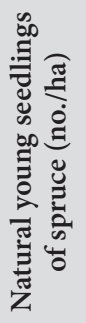 } & $\stackrel{m}{v}$ & $\begin{array}{l}\infty \\
\infty \\
\dot{n}\end{array}$ & $\begin{array}{l}\text { S్ } \\
\stackrel{+}{\sim}\end{array}$ & $\begin{array}{l}\stackrel{10}{\pi} \\
\underset{1}{i}\end{array}$ & $\underset{\infty}{\infty}$ & $\underset{0}{+1}$ & $\stackrel{\overbrace{}}{\tilde{0}}$ & 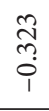 & $\begin{array}{l}n \\
8 \\
0 \\
0 \\
i\end{array}$ & $\begin{array}{l}10 \\
0 \\
0 \\
0\end{array}$ & $\begin{array}{l}\hat{8} \\
\dot{0} \\
\dot{0}\end{array}$ & $\begin{array}{l}\text { ô } \\
\text { o. } \\
\dot{i}\end{array}$ & $\begin{array}{l}\tilde{L} \\
\tilde{O} \\
0 \\
\dot{1}\end{array}$ & $\begin{array}{l}\text { m. } \\
\dot{0} \\
\dot{0}\end{array}$ & 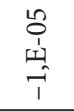 & $\begin{array}{l}8 \\
1 \\
1 \\
1 \\
0 \\
1 \\
1\end{array}$ \\
\hline & $\stackrel{\text { in }}{v}$ & $\begin{array}{l}\vec{\infty} \\
\infty \\
\dot{n}\end{array}$ & 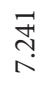 & $\begin{array}{l}\text { ๖ } \\
\text { ì } \\
\text { i }\end{array}$ & $\underset{\substack{+\infty}}{+}$ & $\overrightarrow{\widetilde{f}}$ & $\stackrel{+}{\text { m. }}$ & 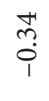 & $\begin{array}{l}n \\
0 \\
0 \\
0 \\
1\end{array}$ & $\begin{array}{l}10 \\
0 \\
0 \\
0\end{array}$ & $\begin{array}{l}\hat{8} \\
\text { o. } \\
\dot{0}\end{array}$ & $\begin{array}{l}\text { mo } \\
\dot{0} \\
\dot{\rho}\end{array}$ & $\begin{array}{l}\tilde{C} \\
0 \\
\dot{0}\end{array}$ & mo̊ & $\begin{array}{l}8 \\
1 \\
1 \\
\text { 1 } \\
1\end{array}$ & $\begin{array}{l}8 \\
\text { 1 } \\
1 \\
\text { 1 } \\
1\end{array}$ \\
\hline & $\vec{v}$ & $\begin{array}{l}\stackrel{2}{0} \\
\stackrel{\infty}{+} \\
+\end{array}$ & 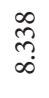 & 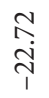 & $\stackrel{m}{\stackrel{m}{a}}$ & $\begin{array}{l}\text { ఫે } \\
\text { in }\end{array}$ & 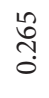 & 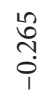 & $\begin{array}{l}\text { \& } \\
\text { o } \\
i\end{array}$ & $\begin{array}{l}n \\
0 \\
0 \\
0\end{array}$ & $\begin{array}{l}\infty \\
8 \\
0 \\
\end{array}$ & $\begin{array}{l}\text { ô } \\
\text { ơ } \\
\text { i }\end{array}$ & స̂. & \%̊̊ & $\begin{array}{l}8 \\
0 \\
1 \\
1 \\
1 \\
1 \\
1\end{array}$ & $\begin{array}{l}0 \\
0 \\
1 \\
11 \\
\text { 1) } \\
1\end{array}$ \\
\hline \multirow{3}{*}{ 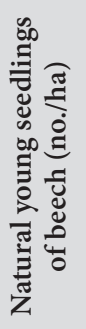 } & $\stackrel{m}{v}$ & $\stackrel{9}{\infty}$ & \begin{tabular}{l} 
To \\
\multirow{1}{*}{} \\
$i$ \\
1
\end{tabular} & $\begin{array}{l}\text { L } \\
1+1 \\
0 \\
0\end{array}$ & \begin{tabular}{l}
+ \\
\multirow{0}{0}{} \\
$i$ \\
$i$
\end{tabular} & 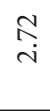 & $\begin{array}{l}+ \\
8 \\
0 \\
i \\
1\end{array}$ & 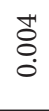 & $\overrightarrow{8}$ & $\begin{array}{l}\overline{8} \\
\dot{0} \\
\dot{1}\end{array}$ & $\tilde{o}_{0}^{0}$ & $\begin{array}{l}m \\
8 \\
\dot{0} \\
1\end{array}$ & $\begin{array}{l} \\
\\
0 \\
0 \\
1\end{array}$ & 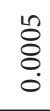 & 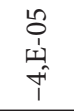 & 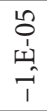 \\
\hline & $\stackrel{\text { in }}{v}$ & 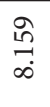 & 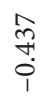 & 굴 & $\begin{array}{l}\stackrel{\text { fे }}{\infty} \\
\text { i }\end{array}$ & 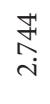 & $\begin{array}{l}0 \\
\dot{0} \\
\dot{0} \\
1\end{array}$ & $\begin{array}{l}\text { oे } \\
\dot{0}\end{array}$ & $\overrightarrow{8}$ & $\begin{array}{l}\overrightarrow{8} \\
0 \\
\dot{0}\end{array}$ & $\stackrel{0}{0}$ & $\begin{array}{l}\text { no } \\
\dot{0} \\
\dot{\varphi}\end{array}$ & $\begin{array}{l}\text { H' } \\
8 \\
0 \\
0\end{array}$ & $\begin{array}{l}\text { Ln } \\
8 \\
8 \\
0\end{array}$ & $\begin{array}{l}\text { L } \\
\text { 1 } \\
\text { I } \\
\text { † }\end{array}$ & 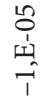 \\
\hline & $\vec{v}$ & $\stackrel{\widehat{\omega}}{\underset{\infty}{\infty}}$ & 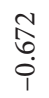 & \begin{tabular}{l} 
Ln \\
\multirow{f}{0}{} \\
0
\end{tabular} & $\begin{array}{l}\underset{\mathbb{J}}{\mathbb{S}} \\
\stackrel{i}{i}\end{array}$ & $\begin{array}{l}\vec{F} \\
\text { i } \\
i\end{array}$ & $\begin{array}{l}N \\
0 \\
0 \\
1\end{array}$ & $\stackrel{N}{0}$ & 官 & $\begin{array}{l}\overrightarrow{8} \\
\dot{0} \\
0\end{array}$ & $\begin{array}{l}\text { Oó } \\
\text { o. }\end{array}$ & $\begin{array}{l}m \\
\dot{8} \\
\dot{0}\end{array}$ & 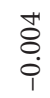 & $\vec{\circ}$ & $\begin{array}{l}\text { 노 } \\
0 \\
1 \\
\text { ب1 } \\
\text { + }\end{array}$ & $\begin{array}{l}\text { 농 } \\
1 \\
1 \\
\\
-1\end{array}$ \\
\hline \multirow{3}{*}{ 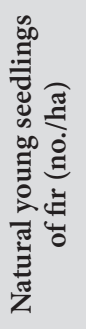 } & $\stackrel{m}{v}$ & $\begin{array}{l}D \\
\infty \\
\infty \\
\infty\end{array}$ & $\begin{array}{l}\stackrel{2}{\circ} \\
\stackrel{\hat{~}}{1}\end{array}$ & $\stackrel{0}{\stackrel{0}{\longrightarrow}}$ & $\underset{\sim}{\stackrel{H}{n}}$ & $\stackrel{\sim}{\sigma}$ & $\begin{array}{l}\hat{a} \\
\text { o. }\end{array}$ & $\begin{array}{l}\hat{a} \\
\hat{0} \\
\dot{1}\end{array}$ & $\begin{array}{l}+ \\
8 \\
0\end{array}$ & $\overrightarrow{8}$ & $\begin{array}{l}\text { mo } \\
\dot{0} \\
\dot{1}\end{array}$ & $\begin{array}{l}\overline{8} \\
\dot{0} \\
\dot{1}\end{array}$ & $\begin{array}{l}m \\
\delta \\
\dot{\rho}\end{array}$ & 용 & $\begin{array}{l}\text { L } \\
\text { 1 } \\
\text { 11 } \\
\text { F̂ }\end{array}$ & 1 \\
\hline & $\begin{array}{l}\text { in } \\
v\end{array}$ & $\underset{\infty}{\stackrel{\sim}{N}}$ & $\begin{array}{l}\infty \\
\infty \\
\infty \\
i \\
i\end{array}$ & $\begin{array}{l}\infty \\
\stackrel{0}{-1} \\
i \\
\end{array}$ & $\stackrel{\infty}{\stackrel{\infty}{\infty}}$ & $\begin{array}{l}\infty \\
\infty \\
\infty \\
0\end{array}$ & $\begin{array}{l}\hat{0} \\
\stackrel{0}{0}\end{array}$ & $\begin{array}{l}\hat{0} \\
\stackrel{-}{0} \\
\text { i }\end{array}$ & $\begin{array}{l}\text { ô } \\
\dot{0}\end{array}$ & $\begin{array}{l}\overrightarrow{8} \\
\dot{0}\end{array}$ & $\begin{array}{l}\text { o̊ } \\
0 \\
\dot{0}\end{array}$ & $\begin{array}{l}\overrightarrow{8} \\
\dot{0} \\
\dot{1}\end{array}$ & 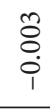 & 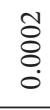 & $\begin{array}{l}\text { L } \\
\text { 1 } \\
\text { I } \\
\text { † }\end{array}$ & 1 \\
\hline & $\vec{v}$ & $\begin{array}{l}\infty \\
\infty \\
\infty \\
\infty\end{array}$ & $\begin{array}{l}\stackrel{N}{N} \\
\stackrel{i}{i}\end{array}$ & $\begin{array}{l}\infty \\
\infty \\
\stackrel{\infty}{0} \\
\hat{i}\end{array}$ & $\begin{array}{l}\stackrel{\mathfrak{n}}{n} \\
\stackrel{n}{n}\end{array}$ & $\begin{array}{l}0 \\
\stackrel{0}{0} \\
0\end{array}$ & 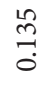 & $\begin{array}{l}m \\
\stackrel{n}{7} \\
\stackrel{0}{0}\end{array}$ & $\begin{array}{l}0 \\
0 \\
0\end{array}$ & $\overrightarrow{8}$ & $\begin{array}{l}\text { ô } \\
\dot{0} \\
\dot{\varphi}\end{array}$ & $\begin{array}{l}\overrightarrow{8} \\
\dot{0} \\
\dot{0}\end{array}$ & $\begin{array}{l}0 \\
8 \\
0 \\
\dot{1}\end{array}$ & $\begin{array}{l}\text { ô } \\
8 \\
0 \\
0\end{array}$ & $\begin{array}{l}\text { Lo } \\
1 \\
\text { I } \\
+1\end{array}$ & 1 \\
\hline 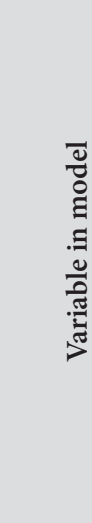 & & 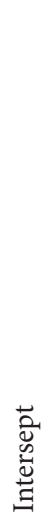 & 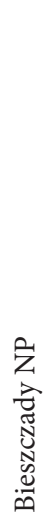 & 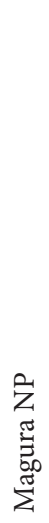 & 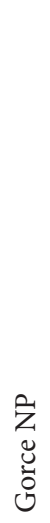 & 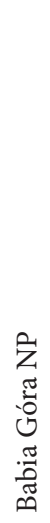 & 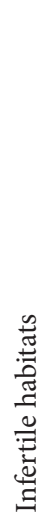 & 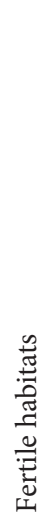 & 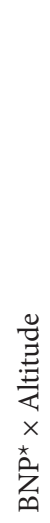 & 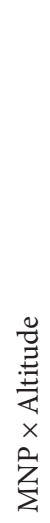 & 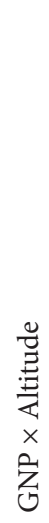 & 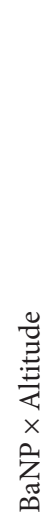 & 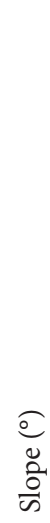 & 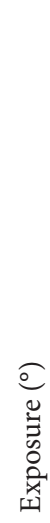 & 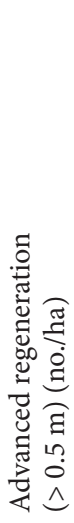 & 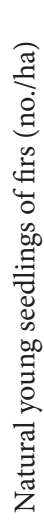 \\
\hline
\end{tabular}




\begin{tabular}{|c|c|c|c|c|c|c|c|c|c|c|c|}
\hline \multirow{3}{*}{ 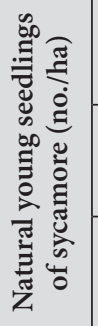 } & $\stackrel{m}{v}$ & 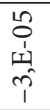 & $\begin{array}{l}+ \\
0 \\
1 \\
+1 \\
+1\end{array}$ & 1 & 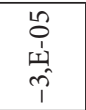 & 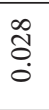 & $\begin{array}{l}0 \\
\vdots \\
0 \\
\\
1\end{array}$ & 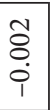 & $\begin{array}{l}\tilde{a} \\
\vdots \\
0\end{array}$ & 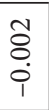 & ટે \\
\hline & $\stackrel{n}{v}$ & 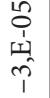 & 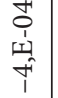 & 1 & $\begin{array}{l}\text { Ln } \\
0 \\
\text { 11 } \\
\text { m) } \\
1\end{array}$ & $\begin{array}{l}\infty \\
\stackrel{\infty}{0} \\
0 \\
0\end{array}$ & $\begin{array}{l}\tilde{m} \\
\tilde{0} \\
\dot{\varphi}\end{array}$ & $\begin{array}{l}20 \\
8 \\
0 \\
\dot{\varphi} \\
1\end{array}$ & $\begin{array}{l}\infty \\
\stackrel{0}{0} \\
\dot{0}\end{array}$ & $\begin{array}{l}0 \\
\text { İ } \\
\stackrel{0}{i}\end{array}$ & $\begin{array}{l}\stackrel{H}{0} \\
\stackrel{0}{0}\end{array}$ \\
\hline & $\vec{v}$ & 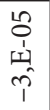 & \begin{tabular}{l|}
+4 \\
0 \\
1 \\
m \\
0 \\
1
\end{tabular} & 1 & 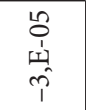 & $\begin{array}{l}\cong \\
\Xi \\
0\end{array}$ & 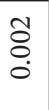 & $\begin{array}{l}\tilde{8} \\
\stackrel{8}{0} \\
\dot{1}\end{array}$ & $\begin{array}{l}n \\
\stackrel{8}{8} \\
\dot{9} \\
1\end{array}$ & $\begin{array}{l}\hat{\infty} \\
\text { o } \\
\dot{0} \\
1\end{array}$ & 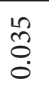 \\
\hline \multirow{3}{*}{ 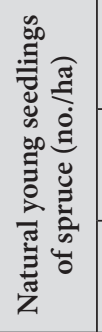 } & $\stackrel{m}{v}$ & $\begin{array}{l}2 \\
\text { oे } \\
\text { i } \\
\text { की } \\
1\end{array}$ & 1 & 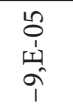 & 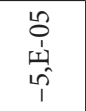 & \begin{tabular}{l}
$\infty$ \\
\multirow{5}{*}{} \\
0 \\
0
\end{tabular} & $\begin{array}{l}\text { mo } \\
0 \\
\dot{\varphi} \\
\text { i }\end{array}$ & $\begin{array}{l}1 \\
\stackrel{8}{0} \\
\dot{0}\end{array}$ & $\begin{array}{l}+ \\
8 \\
0 \\
\dot{i} \\
1\end{array}$ & $\begin{array}{l}\stackrel{+}{0} \\
\stackrel{0}{0} \\
0\end{array}$ & $\begin{array}{l}\vec{b} \\
\dot{0} \\
1\end{array}$ \\
\hline & $\stackrel{n}{v}$ & 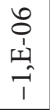 & 1 & 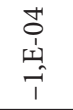 & $\begin{array}{l}\text { L } \\
0 \\
1 \\
1 \\
1 \\
1 \\
1\end{array}$ & $\begin{array}{l}\text { I্ } \\
\text { ㅁ. }\end{array}$ & $\begin{array}{l}+1 \\
\stackrel{0}{0} \\
0\end{array}$ & $\begin{array}{c}\vec{\sigma} \\
\stackrel{0}{0} \\
0\end{array}$ & 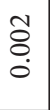 & $\begin{array}{l}\text { İ } \\
\text { Oें } \\
\text { i }\end{array}$ & $\begin{array}{l}\stackrel{\infty}{\sim} \\
\underset{0}{0} \\
i\end{array}$ \\
\hline & $\vec{v}$ & $\begin{array}{l}\hat{o} \\
\text { iे } \\
\text { th } \\
\hat{i}\end{array}$ & 1 & $\begin{array}{l}n \\
0 \\
1 \\
1 \\
\hat{i} \\
\hat{1}\end{array}$ & $\begin{array}{l}0 \\
0 \\
1 \\
1 \\
0 \\
0 \\
1\end{array}$ & $\begin{array}{l}\text { ஜे } \\
\text { oे }\end{array}$ & 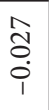 & $\begin{array}{l}+ \\
\stackrel{0}{0} \\
\vdots\end{array}$ & $\begin{array}{l}\text { ⿵े丶 } \\
0 \\
0 \\
i\end{array}$ & $\begin{array}{l}\frac{N}{n} \\
\frac{0}{0} \\
1\end{array}$ & $\begin{array}{l}\hat{0} \\
\stackrel{0}{0}\end{array}$ \\
\hline \multirow{3}{*}{ 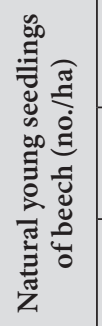 } & $\stackrel{m}{v}$ & 1 & $\begin{array}{l}n \\
0 \\
1 \\
1 \\
\hat{1} \\
1\end{array}$ & 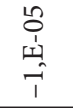 & 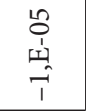 & 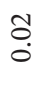 & $\begin{array}{l}\tilde{8} \\
\stackrel{0}{0} \\
\stackrel{1}{1}\end{array}$ & $\begin{array}{l}\tilde{\delta} \\
\dot{0} \\
i\end{array}$ & $\overrightarrow{8}$ & $\begin{array}{l}\hat{\circ} \\
\text { : } \\
\dot{1}\end{array}$ & $\begin{array}{l}\text { Lे } \\
\text { Oे } \\
\dot{0} \\
1\end{array}$ \\
\hline & $\stackrel{i n}{v}$ & 1 & $\begin{array}{l}20 \\
0 \\
1 \\
1 \\
-1 \\
1\end{array}$ & $\begin{array}{l}20 \\
0 \\
1 \\
1 \\
1 \\
1 \\
1\end{array}$ & $\begin{array}{l}20 \\
0 \\
1 \\
1 \\
1 \\
1 \\
1\end{array}$ & $\stackrel{\sigma}{0}$ & $\begin{array}{l}+4 \\
8 \\
0 \\
0 \\
1\end{array}$ & $\begin{array}{l}\overrightarrow{8} \\
0 \\
i \\
1\end{array}$ & $\begin{array}{l}\stackrel{0}{0} \\
\vdots \\
0\end{array}$ & 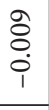 & \begin{tabular}{l}
$\infty$ \\
\multirow{2}{0}{} \\
$\stackrel{0}{0}$ \\
1
\end{tabular} \\
\hline & $\vec{v}$ & 1 & $\begin{array}{l}0 \\
0 \\
1 \\
-1 \\
-1 \\
T\end{array}$ & 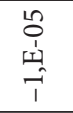 & 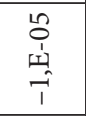 & 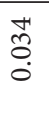 & $\begin{array}{l}\infty \\
8 \\
0 \\
1\end{array}$ & $\begin{array}{l}n \\
0 \\
\dot{0} \\
1\end{array}$ & $\begin{array}{l}\stackrel{1}{*} \\
\ddot{0} \\
\dot{0}\end{array}$ & 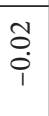 & \begin{tabular}{l}
$\infty$ \\
\multirow{5}{*}{} \\
0 \\
$\dot{\varphi}$
\end{tabular} \\
\hline \multirow{3}{*}{ 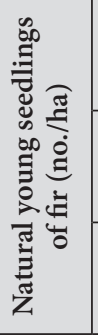 } & $\stackrel{m}{v}$ & 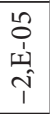 & $\begin{array}{l}0 \\
0 \\
1 \\
\text { 11 } \\
\hat{1} \\
1\end{array}$ & $\begin{array}{l}8 \\
0 \\
1 \\
1 \\
1 \\
1\end{array}$ & 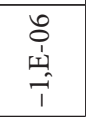 & $\begin{array}{l}\infty \\
\stackrel{\infty}{0} \\
0\end{array}$ & $\begin{array}{l}\overrightarrow{8} \\
\dot{0} \\
\dot{1}\end{array}$ & $\begin{array}{l}0 \\
\tilde{8} \\
0 \\
i\end{array}$ & $\begin{array}{l}\hat{8} \\
\dot{0}\end{array}$ & $\begin{array}{l}\text { I্ } \\
\text { ○े } \\
\text { i }\end{array}$ & $\begin{array}{l}\overrightarrow{8} \\
8 \\
0 \\
0\end{array}$ \\
\hline & $\stackrel{i n}{v}$ & 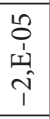 & 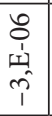 & 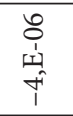 & 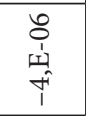 & $\begin{array}{l}\stackrel{2}{0} \\
0 \\
0\end{array}$ & $\begin{array}{l}8 \\
0 \\
0 \\
\dot{1} \\
1\end{array}$ & $\begin{array}{l}\tau \\
\tilde{o} \\
0\end{array}$ & $\begin{array}{l}\stackrel{\partial}{0} \\
\dot{0}\end{array}$ & $\begin{array}{l}20 \\
0 \\
0 \\
0 \\
1\end{array}$ & $\begin{array}{l}20 \\
0 \\
0 \\
0 \\
1\end{array}$ \\
\hline & $\vec{v}$ & 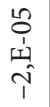 & $\begin{array}{c}0 \\
0 \\
1 \\
11 \\
\hat{1} \\
\hat{1}\end{array}$ & $\begin{array}{l}8 \\
0 \\
1 \\
1 \\
\text { pr } \\
1\end{array}$ & 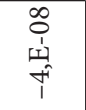 & 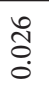 & $\begin{array}{l}\text { oे } \\
\dot{0} \\
\dot{0}\end{array}$ & $\begin{array}{l}\vec{\circ} \\
\stackrel{0}{0}\end{array}$ & $\begin{array}{l}\infty \\
0 \\
0 \\
0 \\
i \\
1\end{array}$ & 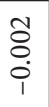 & 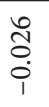 \\
\hline \multicolumn{2}{|c|}{ 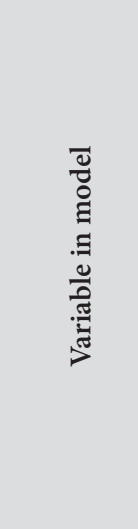 } & 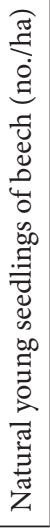 & 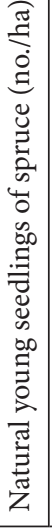 & 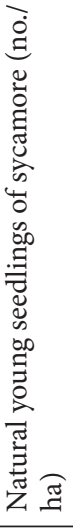 & 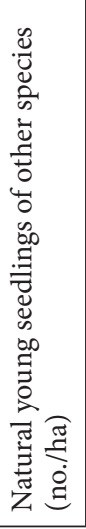 & 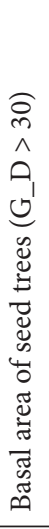 & 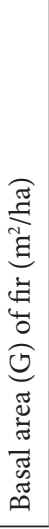 & 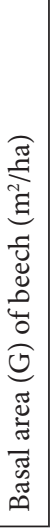 & 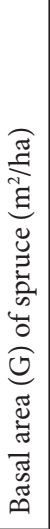 & 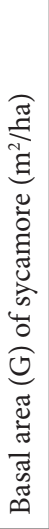 & 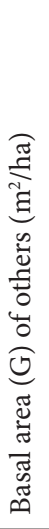 \\
\hline
\end{tabular}


Only parameters of the following independent variables were interpreted: basal area of seed trees of a given species (G_D > 30) and basal area of all trees of a given species in a stand $(G)$. Only statistically significant parameters of these variables were interpreted in the models (see Figs. 1 and 2). In statistical tests, the null hypothesis was rejected at $\mathrm{p} \leq 0.05$.

\section{Results}

The parameters of the variables in the models are given in Table 3. The variable that most determined the number of natural young seedlings was the location of the plot in a given park. Relatively high parameters were also found in the case of the "habitat fertility" variable. Infertile habitats promoted the regeneration of fir and spruce, while fertile promoted the regeneration of beech and sycamore.

The effect of the basal area of seed trees of a given species on the number of its natural young seedlings

On all spatial scales (11, 5, 3 metres), the model parameter values were positive. A greater effect of basal area of seed trees was found in the case of spruce (Gpi_D > 30) and sycamore $\left(\mathrm{Gac} \_D>30\right)$ than in the case of fir $\left(\mathrm{Gab} \_D>30\right)$ and beech $\left(\mathrm{Gfa} \_\mathrm{D}>30\right)$. The highest parameter values were found on the scale of 11 meters (Fig. 1).

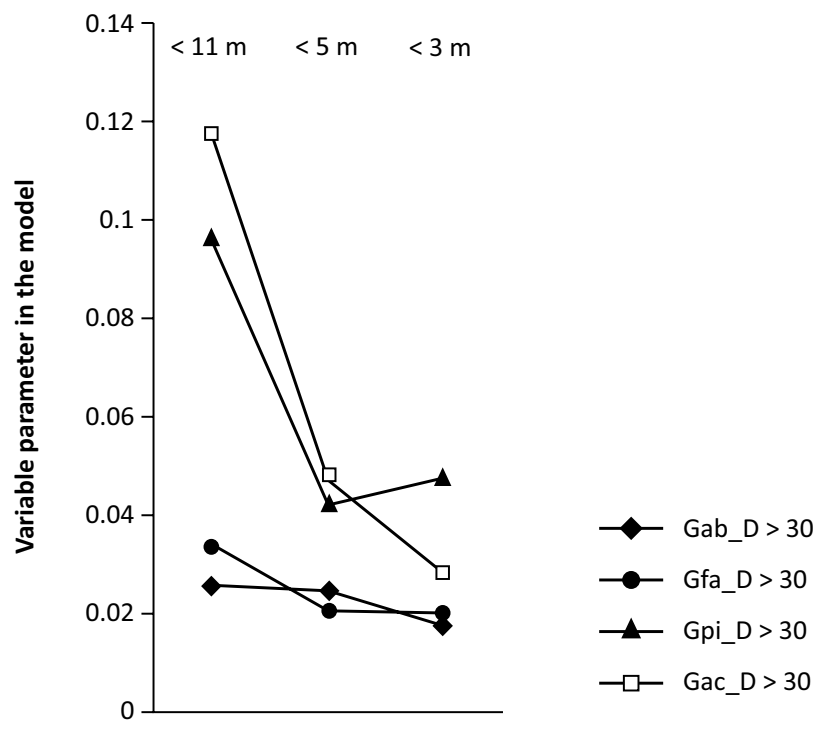

Fig. 1. Variable parameters of the basal area of seed trees $\left(G \_D>30\right)$ of fir $\left(G a b \_D>30\right)$, beech (Gfa_D > 30), spruce (Gpi_D > 30), sycamore (Gac_D > 30) in the analysed models, $<11 \mathrm{~m},<5 \mathrm{~m},<3 \mathrm{~m}$ - radius of a circle (scale) in the model 
The effect of the basal area of species in the stand on the number of natural young seedlings of the analysed species

Number of natural young seedlings of fir

The largest positive parameter values were associated with the variable "basal area of spruce (Gpi)" on the scale of $5 \mathrm{~m}$ and $3 \mathrm{~m}$ (Fig. 2). On the scale of $11 \mathrm{~m}$ and $5 \mathrm{~m}$, very small positive parameters were obtained by the variable "basall area of beech (Gfa)". Other species formed microhabitats limiting the number of natural young seedlings of fir. The variable "basal area of other trees (Gother)" was the most restrictive on the scale of $11 \mathrm{~m}$, and on the scale of $3 \mathrm{~m}$ it was not a significant component of the model. At the smallest scale, the largest negative values of the model parameter were found for sycamore (Gac). The parameter of the variable "basal area of fir (Gab)" had a negative sign on all scales.

Number of natural young seedlings of beech

All species except spruce (Gpi) had negative parameter values in models, regardless of scale (Fig 2). The parameters of the variable "basal area of spruce" were positive. They had the highest value on the scale of 11 meters. The impact of the basal area of fir (Gab) and beech (Gfa) was negative but on the scale of 5 meters it approached neutrality.

Number of natural young seedlings of spruce

Model parameters indicate a positive impact of beech (Gfa) on the number of natural young seedlings of spruce in the three analysed scales (Fig. 2). The highest positive parameter value was found in the range of 5 meters. On this scale, the impact of spruce (Gpi) and fir (Gab) also changed from negative to positive. The impact of beech in the stand on the number of natural young seedlings of spruce was similar to the effect of spruce in the stand on the number of natural young seedlings of beech. The smallest scale $(3 \mathrm{~m})$ revealed a positive impact of the basal area of sycamore (Gac) on the number of natural young seedlings of spruce.

\section{Number of natural young seedlings of sycamore}

The sign and values of the model parameters indicate a beneficial impact of the basal area of the species defined as "other" (Fig. 2) on the number of natural young seedlings of sycamore. On the 5 and 3 metre scale, a positive influence of basal area of spruce was also revealed, the model parameter for this variable (Gpi) obtained the highest value on the 3 metre scale. The parameter of the variable "basal area of sycamore (Gac)" had a negative sign on all scales.

The analysis of Figure 2 suggests that the share of plots in which regeneration was found should be higher in the set of plots on which tree species that positively affect the number of this regeneration grow, in comparison to the set where these species are not present. The analysis of Figure 3 shows that in the case of fir, beech and spruce it is just the opposite. In the set of plots in which the positively affecting species grew in the stand, the plots without natural young seedlings (zero) were majority. 
Similarly, the number of plots with few and numerous natural young seedlings was higher in the pool of plots without the positively affecting species. The presented statements seem to contradict the conclusions resulting from the analysis of Figure 2. A certain convergence with the indications of the models was shown only by the number of plots with natural young seedlings of sycamore, in which other species grow in the stand. In the set of plots where they are present, there are less zero plots, and more with few $\left(\leq 1 \mathrm{no} . / \mathrm{m}^{2}\right)$ and numerous $\left(>1 \mathrm{no} . / \mathrm{m}^{2}\right)$ natural young seedlings of sycamore.
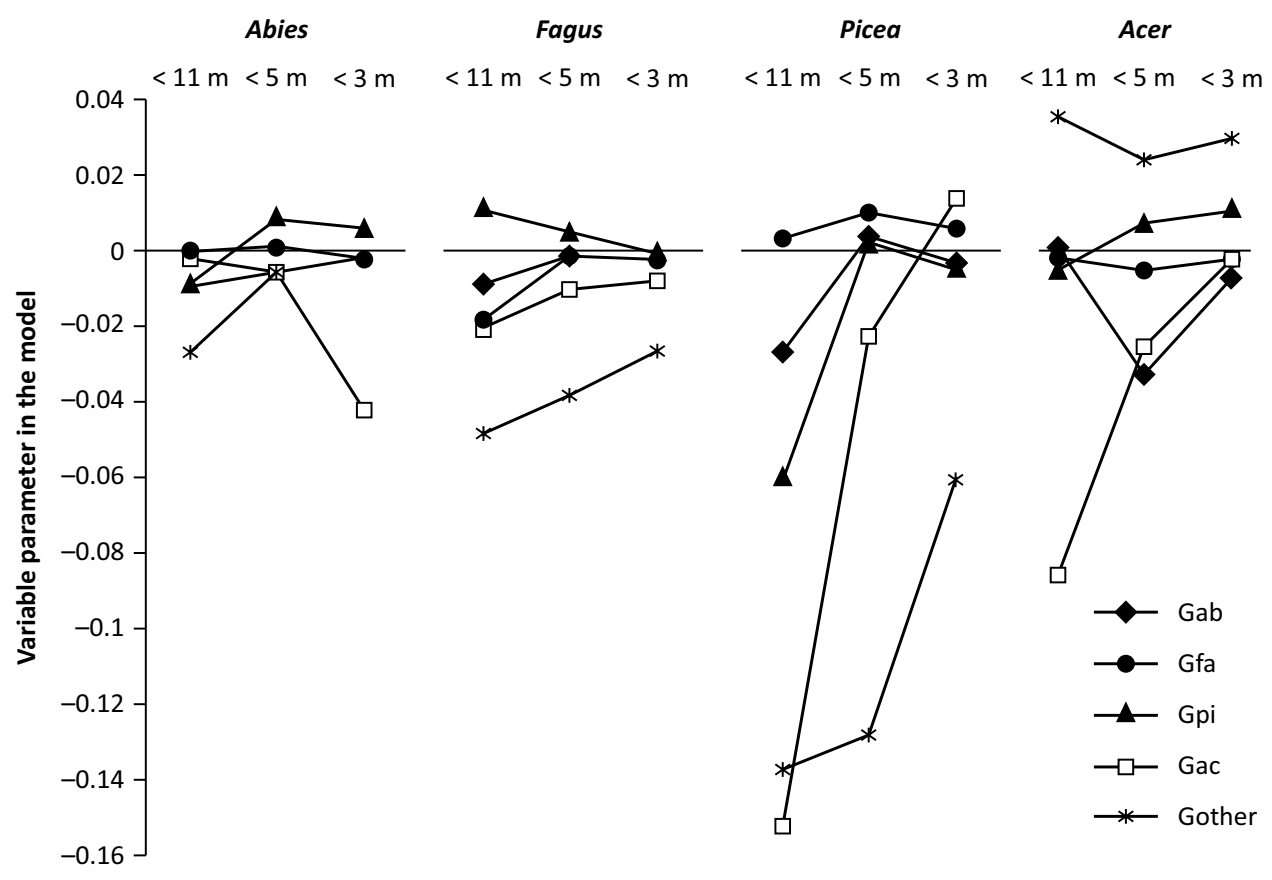

Fig. 2. Parameters of the variable "basal area $(G)$ of fir (Gab), beech (Gfa), spruce (Gpi), sycamore (Gac_D > 30) and other species (Gother)" in the analysed models

A comparison of the average numbers of natural young seedlings in the set of plots where positively affecting species are present with plots where these species are not present (Fig. 4) confirms the relationships indicated by generalised models (see Fig. 2). Stands where positively influencing species, natural young seedlingsof fir, beech, and sycamore were more numerous. For spruce, a similar relationship can be observed only in the case of beech as a positively affecting species in the plots with numerous natural young seedlings. 


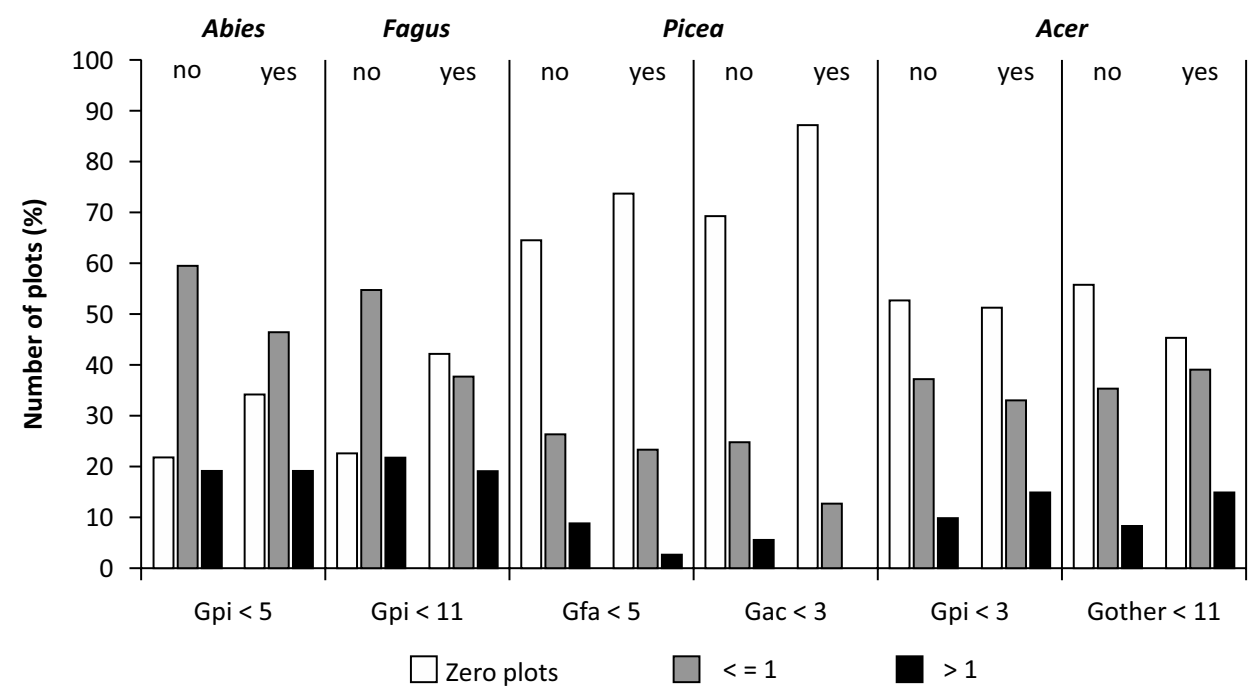

Fig. 3. The share of zero plots, plots with a small number of natural young seedlings ( $\leq 1$ no./ $\mathrm{m}^{2}$ ), and plots with a large number of natural young seedlings $\left(>1 \mathrm{no} . / \mathrm{m}^{2}\right)$ in the set of plots, where positively affecting species are present ("yes"), and such species are not present ("no") in the stand. The horizontal axis indicates the species (and impact radii) with the highest parameters in the analysed models (Fig. 1)

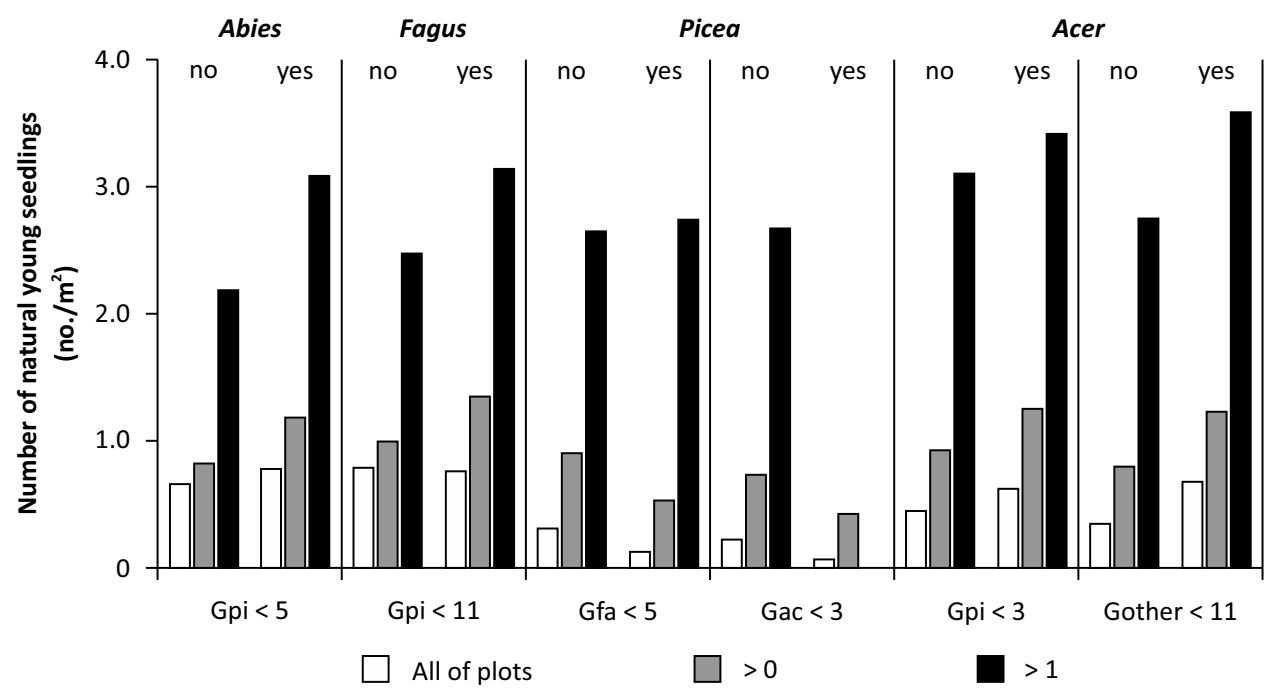

Fig. 4. The average number of natural young seedlings in: all plots, set of plots excluding zero plots, and plots with numerous natural young seedlings $\left(>1 \mathrm{no} . / \mathrm{m}^{2}\right)$, where the positively affecting species is not present ("no") and where it is present ("yes"). The horizontal axis indicates the species (and impact radii) with the highest parameters in the analysed models (Fig. 2) 


\section{Discussion and conclusions}

A positive impact of basal area of potential seed trees of a given species on the number of the species' regeneration was found. A larger basal area of seed trees means a greater seed deposition for a given species. Similar results were obtained by other authors (Paluch and Jastrzębski 2013, Paluch et al. 2016), however, as shown by Paluch et al. (2019) the increase in the number of regeneration of a given species is not directly proportional to the increase of the number of seed trees in the stand. A significantly greater impact of basal area of seed trees on the number of regeneration of a given species (higher parameters in the models) was observed in the case of spruce and sycamore than in the case of shade-tolerant species - fir and beech. The highest parameter values were found on the scale of 11 meters.

The effect of species growing in the stand on the number of natural young seedlings depended on the scale $(11,5,3$ meters) in which the phenomenon was observed. Attention is drawn to the regularity associated with the impact of the basal area of all trees of the species (with the DBH at least $7 \mathrm{~cm}$ ) whose number of natural young seedlings was a dependent (response) variable in the model. On the $11 \mathrm{~m} \mathrm{scale}$, this impact has always been negative. It changed as the scale decreased, so in the case of fir, beech and sycamore on the 3-5 m scale it was approaching neutrality, whereas for spruce it was positive on the $5 \mathrm{~m}$ scale. The presented inference requires verification because the change in scale causes the change of parameters of other variables in the model. However, Paluch and Jastrzębski (2013) also indicated the impact of scale.

The increase in the basal area of other species limited the number of natural young seedlings of fir, beech and spruce. Other species favoured natural young seedlings of sycamore. In other words, other species (mainly pionier species) and sycamore indicated unfavourable ecological niches for regeneration of shade-tolerant species like fir and beech. Spruce regenerated more numerous in the immediate vicinity of sycamore (3-metre scale), but on the 11- and 5-metre scale the occurrence of sycamores limited the number of natural young seedlings of spruce. This probably concerned plots with considerable light access. This observation can be partly related to the results obtained by Paluch et al. (2019). These authors showed that the reduced stand density significantly facilitated the regeneration of spruce in mixed stands with fir and beech but reduced the degree of fir regeneration in mixed stands with beech.

Spruce growing in the stand had a positive impact on the number of natural young seedlings of all analysed species (especially on a 5-metre scale), in contrast to fir which showed no clear positive relationship on any scale with the natural young seedlings of its own or other species. The presence of beech in the stand favoured the regeneration of fir and spruce. For natural young seedlings of spruce, beech had this kind of impact. These relationships confirm the results of studies by other authors (Paluch and Jastrzębski 2013, Paluch et al. 2016, Paluch et al. 2019).

Analysis of frequency of plots without regeneration and with regeneration occurring in stands of different species composition leads to the conclusion that the presence of tree species that positively affect the emergence of natural young seedlings of a given 
species in the stand does not determine the creation of a favourable regeneration niche, in other words it does not guarantee that regeneration will occur in this place. However, if favourable conditions for regeneration occur, then in the places of occurrence of species that positively affect the regeneration of a given species, the natural young seedlings will be more numerous.

\section{References}

Ambroży S. 2002. Analiza wybranych czynników środowiska w odniesieniu do problemu zamierania odnowień jodty pospolitej (Abies alba Mill.). Prace Instytutu Badawczego Leśnictwa. Seria A, 958: 37-59.

Bartkowicz L., Jaworski A., Pach M. 2008. Przypuszczalne mechanizmy zmian udziału jaworu $i$ buka $w$ bieszczadzkich drzewostanach o charakterze pierwotnym. Roczniki Bieszczadzkie, 16: 33-46.

Becker M., Drapier J. 1985. Rôle de l'allélopathie dans les difficultés de régénération du sapin (Abies alba Mill.). Propriétés phytotoxiques des hydrosolubles d'aiguilles de sapin. Oecol. Plant., 5: 347-356.

Bens O., Buczko U., Sieber S., Hüttl R.F. 2006. Spatial variability of O layer thickness and humus forms under different pine beech-forest transformation stages in NE Germany. J. Plant Nutr. Soil Sci. 169: 5-15.

Bernadzki E. 2008. Jodła pospolita. Monografia z serii „Drzewa polskich lasów”. PWRiL, Warszawa.

Brzeziecki B. 2000. Strategie życiowe gatunków drzew leśnych. Sylwan, 144, 8: 5-14.

Bigelow S.W., Canham C.D. 2015. Litterfall as a niche construction process in a northern hardwood forest. Ecosphere, 6: 1-14.

Buck J.R., St Clair S.B. 2014. Stand composition, proximity to overstory trees and gradients of soil moisture influence patterns of subalpine fir seedling emergence and survival. Plant Soil, 381: $61-70$.

Cline E., Vinyard B., Edmonds R. 2007. Spatial effects of retention trees on mycorrhizas and biomass of Douglas-fir seedlings. Can. J. For. Res., 37: 430-438.

Dobrowolska D. 2013. Wzrost i przeżywalność jodły pospolitej (Abies alba Mill.) w zależności od gatunku osłaniajacego na terenie Karkonoskiego Parku Narodowego. Sylwan, 157(3): 197-203.

Grebenc T., Christensen M., Vilhar U., Čater M., Martín M.P., Simončič P., Kraigher H. 2009. Response of ectomycorrhizal community structure to gap opening in natural and managed temperate beech-dominated forests. Can. J. For. Res., 39: 1375-1386.

Hofmeister Š., Svoboda M., Souček J., Vacek S. 2008. Spatial pattern of Norway spruce and silver fir natural regeneration in uneven-aged mixed forests of northeastern Bohemia. J. For. Sci., 54: 92-101.

Jaworski A. 1973. Odnowienie naturalne jodty (Abies alba Mill.) w wybranych zbiorowiskach leśnych Parków Narodowych: Tatrzańskiego, Babiogórskiego i Pienińskiego. Acta Agr. et Silv., Ser. Silv, 13: 21-87.

Jaworski A. 2011. Hodowla lasu. T. III. Charakterystyka hodowlana drzew i krzewów leśnych. PWRiL, Warszawa.

Obrębska-Starklowa B., Hess M., Olecki Z., Trepińska J., Kowanetz L. 1995. Klimat. W: Karpaty Polskie: przyroda, człowiek i jego działalność. Red. J. Warszyńska. Uniwersytet Jagielloński, Kraków. 
Paluch J., Gruba P. 2012. Effect of local species composition on topsoil properties in mixed stands with silver fir (Abies alba Mill.). Forestry, 85: 413-426.

Paluch J., Stępniewska H. 2012. Effect of microsites on the survival, density, and ectomycorrhizal status of shade-tolerant Abies alba regeneration attacked by fungal pathogens. Can. J. For. Res., 42: 720-732.

Paluch J.G., Jastrzębski R. 2013. Natural regeneration of shade-tolerant Abies alba Mill. in gradients of stand species compositions: Limitation by seed availability or safe microsites? Forest Ecology and Management, 307: 322-332.

Paluch J.G., Kołodziej Z., Skrzyszewski J., Bartkowicz L., Gruba P. 2016. Regeneration patterns of the late-successional Abies alba Mill.: inhibition inmonospecific stands and colonization in mixed stands. Annals of Forest Science, 73: 1015-1024.

Paluch J.G., Bartkowicz L., Moser W.K. 2019. Interspecific effects between overstorey and regeneration in small-scale mixtures of three late-successional species in the Western Carpathians (southern Poland). European Journal of Forest Research, 138: 889-905.

Przybylska K., Kucharzyk S. 1999. Skład gatunkowy i struktura lasów Bieszczadzkiego Parku Narodowego. Monografie Bieszczadzkie, 6. Ośrodek Naukowo-Dydaktyczny BdPN, Ustrzyki Dolne.

Stanisz A. 2007. Przystępny kurs statystyki. T. 1-3. Statsoft, Kraków.

Sugiero D. 2005. Jodła pospolita (Abies alba Mill.) w drzewostanach zespołu żyznej buczyny karpackiej (Dentario glandulosae-Fagetum). Sylwan, 11: 45-49.

Szukiel E. 1982. Wplyw przegęszczenia jeleni na odnowienia w lasach bieszczadzkich. Sylwan, 1-3: 41-47.

Szwagrzyk J. 2014. Prawdopodobne zmiany zasięgów występowania gatunków drzewiastych konsekwencje dla hodowli lasu. W: Klimat. Lasy i drewno a zmiany klimatyczne: zagrożenia $i$ szanse. Red. K. Rykowski. Materiały pierwszego panelu ekspertów w ramach prac nad Narodowym Programem Leśnym. Instytut Badawczy Leśnictwa, Sękocin Stary: 1-11.

\section{Acknowledgments}

The authors would like to thank the employees of Bieszczady, Magura, Gorce, and Babia Góra National Parks for providing forest inventory data on permanent monitoring plots.

\section{Financing source}

Publication prepared as part of the NCN - NZ9/00953 grant. "Autoregulatory mechanisms in natural mixed forests - the importance of seed availability and the availability of safe regeneration niches for reproductive success of Abies alba Mill. in the gradient of species composition of forest stands". 


\section{WPŁYW SKŁADU GATUNKOWEGO DRZEWOSTANU NA LICZEBNOŚCI NALOTÓW W WYBRANYCH PARKACH NARODOWYCH KARPAT ZACHODNICH}

\section{STRESZCZENIE}

W pracy wykorzystano dane z 2288 powierzchni próbnych założonych w drzewostanach o ograniczonej ingerencji człowieka na terenie karpackich Parków Narodowych (Bieszczadzkiego, Magurskiego, Gorczańskiego i Babiogórskiego) (tab. 1, 2). Teren badań charakteryzuje pełne spektrum siedliskowe dolnoreglowych drzewostanów zachodniego łuku Karpat. Celem badań była weryfikacja hipotezy, że spośród drzew tworzących drzewostan można wyodrębnić gatunki wpływające pozytywnie bądź negatywnie na liczebność nalotu jodły, buka, świerka i jaworu.

Do analizy wpływu wymienionych zmiennych niezależnych wykorzystano uogólniony model liniowy oparty na rozkładzie Poissona z logarytmiczną funkcją wiążącą.

Zmienną, która w największym stopniu determinowała liczebność nalotów, było położenie powierzchni na terenie danego parku. Relatywnie wysokie parametry stwierdzono również w przypadku zmiennej żyzność siedliska. Siedliska ubogie sprzyjały odnowieniu jodły i świerka, natomiast żyzne - odnowieniu buka i jawora (tab. 3).

Stwierdzono pozytywny wpływ pola przekroju potencjalnych nasienników danego gatunku na liczebność jego odnowień. Wyraźnie większy wpływ pola przekroju nasienników na liczbę odnowień danego gatunku obserwowano w przypadku świerka i jaworu niż w przypadku gatunków cienioznośnych: jodły i buka (ryc. 1). Wzrost pola przekroju wszystkich drzew danego gatunku (o pierśnicy od $7 \mathrm{~cm}$ wzwyż) ograniczał natomiast liczebność nalotów tego gatunku (ryc. 2).

Wpływ gatunków rosnących w drzewostanie na liczebność nalotów zależał od skali (11, 5, 3 metrów), w jakiej zjawisko było obserwowane (ryc. 2).

Wzrost pola przekroju gatunków pionierskich w drzewostanie ograniczał liczebność nalotu jodły, buka i świerka. Gatunki te sprzyjały natomiast pojawianiu się nalotu jaworu. Świerk liczniej odnawiał się w bezpośrednim sąsiedztwie jaworu (skala 3 metrów), ale w skali 11 i 5 metrów występowanie jaworu ograniczało liczebność nalotów świerka (ryc. 2).

Świerk rosnący w drzewostanie dodatnio wpływał na liczebność nalotu wszystkich analizowanych gatunków (zwłaszcza w skali 5 metrów) w przeciwieństwie do jodły, która w żadnej skali nie wykazała wyraźnego pozytywnego związku z liczebnością nalotów, własnego i pozostałych gatunków. Występowanie buka w drzewostanie sprzyjało odnowieniu jodły i świerka. W przypadku nalotów świerka taką rolę odgrywał buk (ryc. 2).

Występowanie w drzewostanie gatunków drzew pozytywnie wpływających na powstanie nalotu danego gatunku nie gwarantuje, że odnowienie w tym miejscu się pojawi (ryc. 3). Jeżeli jednak korzystne warunki do odnowienia zaistnieją, to w miejscach występowania gatunków pozytywnie wpływających odnowienie danego gatunku będzie liczniejsze (ryc. 4).

\section{SŁOWA KLUCZOWE}

odnowienie naturalne $\bullet$ lasy mieszane $\bullet$ Abies $\bullet$ Fagus $\bullet$ Picea $\bullet$ Acer 